\title{
Adaptación y Validación al Español del Cuestionario de Ansiedad por Separación en el Adulto (ASA-27)
}

\author{
Adaptation and Validation in Spanish of the Adult Separation Anxiety Questionnaire \\ (ASA-27)
}

\author{
Antonio Ruiz-García ${ }^{1}$, Óliver Jiménez ${ }^{2}$, Javier Fenollar-Cortés ${ }^{3}$, Josefina Cano-Marín ${ }^{4}$, \\ $\mathrm{M}^{\mathrm{a}}$ Araceli Ojeda-Mora ${ }^{5}$, Ángeles Selva ${ }^{6}$ y Luis Valero-Aguayo ${ }^{7}$
}

\section{Resumen}

Se presenta un estudio de adaptación y validación al español del Cuestionario de Ansiedad por Separación en el Adulto (ASA-27), recientemente incluido en DSM-5. Ha sido traducido y adaptado al español evaluando sus propiedades psicométricas: fiabilidad mediante consistencia interna, análisis factorial y validez concurrente con otros cuestionarios. La versión española de ASA-27 ha mostrado una gran fiabilidad, con una consistencia interna de $\alpha=.92$. Tras el análisis factorial se obtiene un solo factor como en el cuestionario original. En la muestra, aparecen diferencias entre las personas que recibían tratamiento (psiquiátrico y/o psicológico) frente a personas que no. Finalmente, se aporta un baremo en percentiles para un posible diagnóstico del trastorno. Los resultados en esta muestra indican que esta versión en español muestra unas propiedades psicométricas adecuadas para la evaluación de ansiedad por separación en el adulto.

Palabras clave: ansiedad por separación, ASA-27, adultos, cuestionario, estudio instrumental

\begin{abstract}
This is a Spanish adaptation and validation study of the Adult Separation Anxiety Questionnaire (ASA-27), recently included in DSM-5. It has been translated and adapted to Spanish, evaluating its psychometric properties: Internal Consistency Reliability, factor analysis, concurrent validity with other questionnaires. The Spanish version of ASA-27 has shown a great reliability, with an internal consistence of .92 . The factor analysis explains a single factor that groups all the items, like the original study. The sample shows differences between people who were receiving psychiatric or psychological treatment and people who were not. Finally, it is given a percentile scale for possible clinical diagnosis. The results in this sample points out that this Spanish version provides a valid and reliable measure for adult separation anxiety.
\end{abstract}

Keywords: separation Anxiety, ASA-27, adults, questionnaire, instrumental study

\footnotetext{
${ }^{1}$ Profesor Sustituto Interino. Doctor en Psicología. Departamento de Psicología, Facultad de Ciencias de la Educación, Universidad de Córdoba. Av. San Alberto Magno, s/n 14071 Córdoba, España. Tel.: +34957218958. Correo: aruiz1@uco.es

${ }^{2}$ Doctorando en Psicología. Facultad de Psicología, Universidad de Málaga. Facultad de Psicología, Campus Teatinos, 29071

Málaga, España. Tel.: +34644477898. Correo: oliverjjimenez@uma.es

${ }^{3}$ Doctor en Psicología. Departamento de Psicología, Universidad Loyola Andalucía. Campus Palmas Altas, 41014 Sevilla, España.

Tel.: +34 955641600- ext 466

${ }^{4}$ Profesor Catedrático de Universidad. Depto Personalidad, Evaluación y Tratamiento Psicológico, Facultad de Psicología. Campus

Teatinos, 29071 Málaga, España. Tel.: 952-132531. Correo: 1valero@uma.es

${ }^{5}$ Psicóloga. Servicio de Atención Psicológica, Facultad de Psicología. Campus Teatinos, 29071 Málaga, España. Tel.: 952-132531.

Correo: jcanom@uma.es

${ }^{6}$ Licenciada en Psicología. Colaboradora honorífica. Facultad de Psicología, Universidad de Málaga. Facultad de Psicología Campus Teatinos, 29071 Málaga, España. Tel.: +34654479232. Correo: ojedamorapsi@gmail.com

${ }^{7}$ Doctora en Psicología. Colaboradora honorífica. Facultad de Psicología. Universidad de Málaga. Facultad de Psicología. Campus Teatinos, 29071 Málaga, España. Tel.: +34654479232. Correo: angelesselva@ yahoo.es
} 


\section{Introducción}

El trastorno de ansiedad por separación (TAS) ha sido un diagnóstico asociado habitualmente a la etapa infantil (Baldwin, Gordon, Abelli, \& Pini, 2016; Bruschi et al., 2012; Gesi et al., 2015; Pini, Abelli, \& Bruschi, 2014a). De hecho, hasta el manual DSM-IV ha sido clasificado en el apartado "Trastornos de comienzo en la infancia, la niñez o adolescencia". Sin embargo, tras las evidencias encontradas de este trastorno en la edad adulta (Bögels, Knappe, \& Clarck, 2013; Manicavasagar \& Silove, 2016), junto al ingente número de publicaciones en las dos últimas décadas tras el primer artículo de Manicavasagar y Silove (1997), sobre el trastorno de ansiedad por separación en la edad adulta (TASA), este diagnóstico ha encontrado su propio espacio en DSM-5 (APA, 2014; Milrod et al., 2014; Schneier et al., 2017). Además, se ha reconocido que puede tener su comienzo en la propia adultez, aunque en una parte de los casos este diagnóstico representa una forma persistente o recurrente del tipo de inicio infantil hasta la vida adulta (González, Ysern, Martorell, Matéu, \& Barreto, 2011; Manicavasagar et al., 2010; Silove \& Rees, 2014). En el "estudio de la Organización Mundial de la Salud (OMS)" el 43\% de los encuestados con trastorno por separación, informaron de su inicio sobre los 20 años (Kessler \& Ustun, 2004; Shear, Jin, Ruscio, Walters, \& Kessler, 2006), encontrándose además que uno de cada tres casos persistieron de la infancia a la edad adulta (Carmassi, Gesi, Massimetti, Shear, \& Dell'Osso, 2015a).

Este cuadro en la adultez se caracteriza por experimentar un exceso de angustia ante la ocurrencia real o la percepción de la separación del hogar o de las principales figuras de apego (Silove et al., 2007), como mudarse de casa de los padres o poner fin a una relación estable de pareja (Mabilia, Di Riso, Liso, \& Bobbio, 2019). Las personas se preocupan por el bienestar o muerte potencial de sus figuras de apego, sobre todo cuando se separan de ellas por la preocupación de conocer su paradero y por la necesidad de sentirse obligados a permanecer en contacto con ellas (Gesi et al., 2015; Pozzi et al., 2014; Posavac \& Posavac, 2017). Produciendo por tanto un desajuste psicológico general (Román, Hodges, Palacios, Moreno, \& Hillman, 2015).

El TASA ha sido asociado en mayor frecuencia a la mujer, con tener un nivel educativo de bajo a medio-alto, un funcionamiento desadaptativo familiar u otras adversidades en la niñez y diversas experiencias de eventos traumáticos en la vida. Estas características de mayores adversidades, mal funcionamiento familiar y eventos traumáticos serían factores precipitantes no solo del inicio en edad pediátrica, sino también del comienzo en la edad adulta de este trastorno (Carmassi et al., 2015a).

Según el National Comorbidity Survey Replication (NCS-R), en los Estados Unidos se encontró un rango de prevalencia vital para el TASA en población general del 6.7\% (Kessler, Pethukova, Sampson, Zaslavsky, \& Wittchen, 2012). Estos datos se obtuvieron mediante evaluación retrospectiva de los síntomas empleando el DSM-IV con la modificación del criterio A en lo relativo especificaciones en los síntomas, edad de inicio y preguntas recientes sobre trastorno de ansiedad por separación del adulto y modificaciones apropiadas a la edad de los pacientes. En el contexto Europeo, atendiendo a criterios DSM-IV evaluados mediante la entrevista CIDI (Silove et al., 2015) se encontró una prevalencia de $4.8 \%$ en la muestra total (rango de .2 a 9.8). Concretamente en algunos países europeos hallaron las siguientes prevalencias: en España un 1.2\%, en Francia un $3.5 \%$, Alemania $2.0 \%$, Portugal un $6.4 \%$ y en Bélgica de un $1.4 \%$, entre otros.

Aunque el TASA parece estar precedido por elevados niveles de ansiedad también en la niñez, son necesarios estudios prospectivos que eliminen los sesgos debidos a la memoria de los informantes, que sería un sesgo característico en los estudios retrospectivos (Bögels et al., 2013). En este mismo estudio encontraron que el $77.5 \%$ de la muestra con TASA comenzó en la edad adulta. Donde el 53\% tenían historia de trastornos del estado de ánimo y el 75\% habían recibido o estaba recibiendo tratamiento de sus problemas emocionales. Asimismo, en muestras clínicas se han encontrado prevalencias estimadas en un rango desde el 20\% al 65\% (Gesi et al., 2015; Shear et al., 2006; Silove \& Rees, 2014). 
El TASA presenta comorbilidad con diversos trastornos (Bruschi et al., 2012; Carmassi et al., 2015b; Gesi et al., 2017; Möller \& Bögels, 2016; Pini et al., 2014b), como los del estado de ánimo, el estrés postraumático, de ansiedad, duelo complicado y trastornos de personalidad (Pozzi et al., 2014). Silove et al. (2010) encontraron que pacientes con TASA con inicio en la infancia presentaron más trastornos de personalidad comórbidos (categorías B y C) que aquellos con el inicio en la edad adulta (Bögels et al., 2013). También se ha hallado comorbilidad con el trastorno de pánico (Fagiolini, Shear, Cassano, \& Frank, 1998; Gesi et al., 2015; Kossowsky et al., 2013; Pini et al., 2010; Silove, Marnane, Wagner, Manicavasagar, \& Rees, 2010) asociado a un marcado deterioro funcional. Manicavasagar, Silove, Curtis y Wagner (2000) encontraron una tasa del $40 \%$ de TASA en pacientes con trastorno de pánico y ansiedad generalizada. Así como comorbilidad entre trastorno bipolar y TASA (Pini et al., 2005), habiéndose encontrado que TASA en la juventud y adultez es significativamente más prevalente en personas con trastorno bipolar tipo I, trastorno de pánico y trastorno depresivo mayor (Costa et al., 2017). Siguiendo a Dell'Osso et al. (2011), el trastorno de ansiedad por separación entre la infancia y la adolescencia, se ha descrito en la población juvenil como antecedente de trastornos de ansiedad y del estado de ánimo en la edad adulta. Encontrando mayor frecuencia de pacientes jóvenes con TASA que presentaban además trastorno de pánico y trastorno bipolar II, frente a aquellos que sólo tenían diagnóstico de trastorno de pánico (Toni et al., 2008). Igualmente, en jóvenes y adultos la ansiedad por separación se ha asociado con trastornos del espectro bipolar $\mathrm{y}$ especialmente al tipo ciclotímico (Perugi \& Akiskal, 2002; Perugi et al., 2012).

La evaluación del TASA se ha caracterizado principalmente por el uso de entrevistas y autoinformes, centrándose en su mayoría en la etapa infanto-juvenil (Bruschi et al., 2012; Manicavasagar, Silove, Wagner, \& Drobny, 2003; Pini et al., 2014a). Para adultos se han empleado medidas retrospectivas de la etapa juvenil, como el Inventario de Síntomas de Ansiedad por Separación (SASI; Silove et al., 1993) que recoge recuerdos en relación a experiencias anteriores a los 18 años. Consta de 15 ítems y cuatro escalas: ansiedad por separación, fobia escolar, angustia por estar lejos de una base segura, y miedo a que sufran daños los miembros de la familia. Presenta una consistencia interna entre $\alpha=.84$ y $\alpha=.88$, y fiabilidad test-retest de .86 a .98 .

En la etapa adulta se ha evaluado el estilo de apego mediante la Entrevista de Apego Adulto de Berkeley (George, Kaplan, \& Main, 1985) y el Cuestionario de Estilo de Apego (Feeney, Noller, \& Hanrahan, 1994). También se ha evaluado el TAS en términos de un rasgo de personalidad. Este es el caso del instrumento Medida de Sensibilidad Interpersonal (IPSM) (Boyce \& Parker, 1989), formado por 36 ítems con una subescala de ansiedad por separación, necesidad de aprobación, timidez, conciencia interpersonal y fragilidad del yo. Habiendo mostrado una consistencia interna entre $\alpha=.55$ y $\alpha=.79$. Además, se han empleado la Entrevista Clínica Estructurada para los Sintomas de Ansiedad por Separación (SCI-SAS) desarrollada para la evaluación mediante criterios DSM-IV-TR del TAS en la infancia, y adaptados para adultos (Cyranowski, Shear, \& Rucci, 2002); y, la Entrevista Estructurada de Ansiedad por Separación en el Adulto (ASA-SI), derivada de los criterios diagnósticos DSM-IV-TR del trastorno infantil adaptada a la edad adulta (Manicavasagar \& Silove, 1997).

A partir de esta entrevista se elaboró una escala autoaplicada, denominada Cuestionario de Ansiedad por Separación en Adultos (ASA-27) con los mismos ítems del ASA-SI aportando además un valor de corte para establecer el diagnóstico (Manicavasagar et al., 2003). Este cuestionario ha sido empleado en diversos estudios habiéndose encontrado relación entre ansiedad por separación y duelo complicado, síntomas maníacos y depresivos (Carmassi et al., 2015b; Dell'Osso et al., 2011). En un estudio posterior, el $70 \%$ de personas con duelo complicado que buscaron ayuda profesional puntuaron en ASA-27 de forma consistente con un cuadro de TASA (Gesi et al., 2017), frente a otros estudios que lo encontraron en un rango del $23 \%$ al $54 \%$ en personas que no buscaban esa ayuda (Manicavasagar et al., 2000; Pini et al., 2010; Silove et al., 2010). 
Debido a la demanda por este tipo de casos en nuestro servicio y la inexistencia de un cuestionario específico para la evaluación de TASA en población española (Fernández-Marcos, 2014; Ruiz \& Rando, 2015), surge la necesidad de su adaptación.

El objetivo general de este estudio es la adaptación del ASA-27 en población española. Este ha sido adaptado en población turca por Dirioz, Alkin, Yemez, Onur y Eminagaoglu (2011), obteniendo una consistencia interna de .93 y fiabilidad test-retest .93.

En cuanto a los objetivos específicos: (a) adaptar el cuestionario ASA-27 a población española; (b) evaluar las propiedades psicométricas, fiabilidad por consistencia interna, y validez convergente con otros instrumentos; (c) proporcionar criterios clínicos para diferenciar personas con y sin trastorno; y (d) determinar posibles diferencias entre grupos de tratamiento, sexo, edad, estudios y tipo de trastorno clínico diagnosticado.

\section{Método}

\section{Participantes}

Han participado 403 personas, siendo una muestra adecuada dentro de la literatura científica. Los participantes fueron 225 mujeres $(55.8 \%) \mathrm{y}$ 178 hombres (44.2\%), con 42.06 años de media $(D T=13.86)$, en un rango entre 22 y 91 años; de ellos 35 (8.7\%) presentaban algún tipo de trastorno psicológico y el $19.6 \%$ afirmaban encontrarse en tratamiento, siendo el $11.9 \%$ médico, el $2.5 \%$ psiquiátrico y el $5.2 \%$ psicológico (trastornos bipolar, depresión, distimia, ansiedad por separación, fobias y obsesiones). Además se dividió la muestra por rangos de edad de 5 años, excepto los menores de 25 y mayores de 65 años, para comparar posteriormente los diferentes grupos de edad.

\section{Instrumentos}

Para obtener la validez concurrente del cuestionario, se han utilizado varios instrumentos que miden constructos similares, como la ansiedad estado-rasgo, el apego y la dependencia emocional:
Tabla 1. Datos sociodemográficos de la muestra

\begin{tabular}{|c|c|c|}
\hline Variables & Frecuencia & $\%$ \\
\hline \multicolumn{3}{|l|}{ Sexo } \\
\hline Hombre & 178 & 44.2 \\
\hline Mujer & 225 & 55.8 \\
\hline \multicolumn{3}{|l|}{ Estado Civil } \\
\hline Soltero/a & 233 & 57.8 \\
\hline Casado/a & 129 & 32 \\
\hline Separado/a & 24 & 6 \\
\hline Viudo & 5 & 1.2 \\
\hline Otros & 11 & 2.7 \\
\hline \multicolumn{3}{|l|}{ Ocupación } \\
\hline Hogar & 25 & 6.2 \\
\hline Paro & 47 & 11.7 \\
\hline Estudiante & 116 & 28.8 \\
\hline Trabajador/a & 146 & 36.2 \\
\hline Autónomo/a & 46 & 11.4 \\
\hline Otra & 20 & 5 \\
\hline \multicolumn{3}{|l|}{ Nivel de estudios } \\
\hline Ninguno & 1 & 0.2 \\
\hline Primaria & 28 & 4.7 \\
\hline Secundaria & 49 & 4.7 \\
\hline Bachillerato & 54 & 14.6 \\
\hline Formación Profesional & 57 & 13.9 \\
\hline Universitario & 184 & 49.9 \\
\hline Posgrado & 44 & 11.9 \\
\hline \multicolumn{3}{|l|}{ Grupo de Edad } \\
\hline Menos de 25 años & 41 & 9.8 \\
\hline Entre 26-30 años & 100 & 24 \\
\hline Entre 31-35 años & 51 & 12.2 \\
\hline Entre 36-40 años & 37 & 8.9 \\
\hline Entre $41-45$ años & 43 & 10.3 \\
\hline Entre 46-50 años & 38 & 9.1 \\
\hline Entre 51-55 años & 37 & 8.9 \\
\hline Entre 56-60 años & 32 & 7.7 \\
\hline Entre 61-65 años & 12 & 2.9 \\
\hline Más de 65 años & 22 & 5.3 \\
\hline \multicolumn{3}{|l|}{ Residencia } \\
\hline Padres & 142 & 35.2 \\
\hline Propia & 164 & 40.7 \\
\hline Vivienda compartida & 57 & 14.1 \\
\hline Vivienda propia & 29 & 7.2 \\
\hline Otros & 8 & 2 \\
\hline \multicolumn{3}{|l|}{ Tratamiento } \\
\hline Ninguno & 323 & 80.1 \\
\hline Médico & 48 & 11.9 \\
\hline Psiquiátrico & 10 & 2.5 \\
\hline Psicológico & 21 & 5.2 \\
\hline \multicolumn{3}{|l|}{ Trastornos psicológicos } \\
\hline No & 368 & 91.3 \\
\hline Sí & 35 & 8.7 \\
\hline
\end{tabular}

Inventario de Ansiedad Estado-Rasgo (STAI; Spielberger, Gorsuch, \& Lushene, 1994). Evalúa la ansiedad en sus constructos estado y rasgo. Presenta 20 ítems con una escala likert de cuatro puntos por subescala. Ha mostrado una fiabilidad de $\alpha=.93$ y $\alpha=.87$, respectivamente en la escala original.

Cuestionario de Apego Adulto (CAA; Melero $\&$ Cantero, 2008). Autoinforme de 40 reactivos con una escala likert de siete puntos, con cuatro 
subescalas: Escala 1, baja autoestima, necesidad de aprobación y miedo al rechazo; Escala 2, resolución hostil de conflictos, rencor y posesividad; Escala 3, expresión de sentimientos y comodidad con las relaciones; Escala 4, autosuficiencia emocional e incomodidad con la intimidad. Presenta una consistencia interna de $\alpha=.86$ para la primera escala, $\alpha=.80$ para la segunda, $\alpha=.77$ para la tercera y $\alpha=.68$ para la cuarta, baremos para población española (Melero \& Cantero, 2008).

Cuestionario de Dependencia Emocional (CDE; Lemos \& Londoño, 2006). Instrumento compuesto por 23 ítems en una escala likert de seis puntos, con seis subescalas: Ansiedad de separación, Expresión afectiva de la pareja, Modificación de planes, Miedo a la soledad, Expresión límite y Búsqueda de atención. Ha mostrado una fiabilidad total de $\alpha=.93$, y de $\alpha=.87, .83, .75, .79, .61$ y .77 para las seis subescalas respectivamente. Ofrece validación y baremación para población colombiana.

Cuestionario de Ansiedad por Separación en el Adulto (ASA-27; Manicavasagar, Silove, Wagner \& Drobny, 2012). Consta de 27 reactivos en escala tipo Likert de cuatro puntos, de una serie de problemas emocionales después de los 18 años de edad. Sus ítems se refieren a: ansiedad y pánico; malestar ante el abandono de personas cercanas o pensar en hacerlo cuando va a trabajar, viajar...; tener pesadillas y preocupación relacionadas con ser abandonado, entre otros. Se compone de un factor general que explica el $45 \%$ de la varianza y ha mostrado un $\alpha=.95$ y fiabilidad test retest de .86 en los estudios originales (Manicavasagar et al., 2003; Manicavasagar et al., 2010).

\section{Procedimiento}

Para la adaptación del cuestionario ASA-27 se llevó acabo la traducción del original en inglés al español por varios expertos con un alto dominio del idioma y del vocabulario empleado en el mismo. Posteriormente la versión en español la evaluaron expertos externos con alto dominio en inglés y realizaron la retrotraducción de esta, nuevamente al inglés. Finalmente, entre la versión retrotraducida y la original no hubo cambios significativos en aspectos formales ni de significado. Los traductores fueron tres nativos españoles con alto nivel de inglés, y los expertos externos fueron dos nativos ingleses conocedores del vocabulario y constructos psicológicos empleados. El cuestionario se aplicó en población general española realizando pasaciones colectivas con grupos de diferentes contextos: universidad, Escuela Oficial de Idiomas, Servicio de Atención Psicológica, centros de psicología y de formación privada. Así como muestreo de bola de nieve, con la instrucción de seleccionar a personas de diferente sexo al suyo y de mayor edad, preferentemente con el objetivo de reclutar muestra más difícilmente accesible. Las pasaciones fueron realizadas en grupo por dos colaboradores formados previamente, explicando en primer lugar el consentimiento informado y a continuación cómo cumplimentar la batería de cuestionarios.

Los participantes fueron informados de su participación voluntaria, y del uso y tratamiento anónimo de sus datos en un consentimiento informado, respetando así las garantías éticas necesarias. Cumplimentando a continuación: STAI E-R, CAA, CDE y ASA-27. La duración aproximada de cada aplicación fue de 30 minutos.

\section{Estrategia de análisis}

Se llevó a cabo un análisis factorial confirmatorio con estimación WLSMV (Meanand Variance-adjusted Weighted Least Squares) para contrastar la estructura factorial original todos los ítems cargando sobre un factor-. Con el fin de explorar la invarianza, se llevaron a cabo análisis factoriales confirmatorios según el sexo. Se excluyeron los casos con valores perdidos. Además, se calculó la chi-cuadrado, el índice de ajuste comparativo (CFI; Comparative Fit Index), el índice de Tucker-Lewis (TLI; Tucker-Lewis Index) y el error cuadrático medio de aproximación (RMSEA; Root-Mean-Square Error of Approximation). Los criterios mínimos para la bondad de ajuste del modelo fueron, TLI y CFI > .90 (Bentler \& Bonett, 1980) y un RMSEA entre .05 y .1 (Browne \& Cudeck, 1992).

A continuación, se calculó el índice de fiabilidad a través del estadístico alfa de Cronbach, considerando aceptables valores por encima de .70 (Nunnally, 1978). Se exploró si la edad de los participantes correlacionaba con la puntuación en ASA-27, con el fin de explorar si 
Tabla 2. Resultado del análisis factorial confirmatorio para la versión española del ASA-27 (ítems resumidos)

\begin{tabular}{|c|c|c|c|}
\hline Ítems resumidos de la escala & $\begin{array}{l}\text { Carga } \\
\text { factorial }\end{array}$ & $\begin{array}{l}\text { Error } \\
\text { estándar }\end{array}$ & $\begin{array}{l}\text { Varianza } \\
\text { residual }\end{array}$ \\
\hline Ítem 1 (Seguridad con personas cercanas) & .415 & .046 & .172 \\
\hline Ítem 2 (Lejanía de casa) & .632 & .051 & .400 \\
\hline Ítem 3 (Objeto de seguridad en el bolso) & .571 & .044 & .326 \\
\hline Ítem 4 (Estrés ante un viaje largo) & 686 & .033 & .470 \\
\hline Ítem 5 (Sueño y pesadillas por separación) & ,707 & .030 & .500 \\
\hline Ítem 6 (Estrés ante viaje de persona cercana) & .739 & .030 & .546 \\
\hline Ítem 7. (Molestia por interrupción de rutina) & .540 & .040 & .291 \\
\hline Ítem 8 (Preocupación por intensidad de relación) & .570 & .038 & .325 \\
\hline Ítem 9 (Dolores o náuseas anticipatorias) & .603 & .039 & .363 \\
\hline Ítem 10 (Habla excesiva para mantener la cercanía) & .590 & .042 & .348 \\
\hline Ítem 11 (Preocupación por otros cuando están ausentes) & .589 & .041 & .346 \\
\hline Ítem 12 (Dificultades de sueño en soledad) & 620 & .036 & .384 \\
\hline Ítem 13 (Mejoría sueño cuando personas cerca) & .586 & .040 & .344 \\
\hline Ítem 14 (Ansiedad por separación o lejanía de personas) & .747 & .029 & .558 \\
\hline Ítem 15 (Pesadillas por lejanía del hogar) & .840 & .034 & .705 \\
\hline Ítem 16 (Preocupación por pensamiento catastrofistas) & .600 & .036 & .360 \\
\hline Ítem 17 (Molestias por cambios de rutina si afecta a relaciones) & .646 & .036 & .417 \\
\hline Ítem 18 (Preocupación por abandono de personas cercanas) & 684 & .031 & .468 \\
\hline Ítem 19 (Mejoría del sueño si hay luces encendidas) & .596 & .051 & .355 \\
\hline Ítem 20 (Evitación de soledad en casa) & .706 & .040 & .499 \\
\hline Ítem 21 (Ansiedad o ataque de ansiedad por lejanía de personas) & .838 & .025 & .702 \\
\hline Ítem 22 (Ansiedad por no contacto telefónico) & .738 & .032 & .545 \\
\hline Ítem 23 (Temor por abandono de alguien cercano) & .635 & .033 & .404 \\
\hline Ítem 24 (Ocurrencia de ataques de pánico ante soledad) & .844 & .028 & .712 \\
\hline Ítem 25 (Preocupación excesiva por pensamientos de daño ajeno) & .710 & .031 & .504 \\
\hline Ítem 26 (Comentarios negativos por habla excesiva) & .397 & .051 & .157 \\
\hline Ítem 27 (Preocupación por problemas ajenos a causa propia) & .589 & .046 & .347 \\
\hline Bondad de ajuste & & & \\
\hline$X^{2}($ g.l. $)$ & \multicolumn{3}{|c|}{$912.9(324)$} \\
\hline Significatividad de $X^{2}$ & \multicolumn{3}{|c|}{$<.001$} \\
\hline CFI & \multicolumn{3}{|c|}{.918} \\
\hline TLI & \multicolumn{3}{|c|}{.911} \\
\hline RMSEA 95\% [CI] & \multicolumn{3}{|c|}{$.067[.062-.072]$} \\
\hline
\end{tabular}

dicha variable debía ser tratada como covariable. Para estudiar la validez convergente, se realizó un análisis correlacional entre la escala ASA-27 adaptada y el resto de las escalas sobre ansiedad, apego y dependencia (STAI E-R, CAA y CDE). Se consideraron correlaciones de .1 como efecto pequeño, .3 como efecto medio y .5 como efecto largo (Cohen, Cohen, West, \& Aiken, 2002). Además, se llevaron a cabo análisis de regresión múltiple con la puntuación en ASA-27 como variable dependiente, y como variables independientes se asumieron las puntuaciones en las diferentes subpruebas empleadas para la validez convergente.

Además, se realizó una prueba $t$ para grupos independientes, comparando los participantes con y sin tratamiento de algún tipo (médico, psiquiátrico y/o psicológico), para comprobar si existían diferencias en ansiedad por separación entre ellos. Finalmente, se elaboró una tabla con la baremación en percentiles de las puntuaciones directas (ver Anexo 2).

\section{Resultados}

El análisis factorial confirmatorio mostró una adecuada bondad de ajuste $\left(\chi^{2}=912.9 ;\right.$ g.l. $=324$; CFI $=.918 ; \quad$ TLI $=.911 ; \quad$ RMSEA $\quad 95 \%[\mathrm{CI}]=.067$ [.062-.072]), donde los ítems mostraron cargas factoriales entre .397 y .844 y varianzas residuales de .17 a .71 (ver Tabla 2). El ajuste del modelo unifactorial fue adecuado tanto para hombres $\left(\chi^{2}=478,3 ;\right.$ g. $l .=324 ;$ CFI=.95; TLI=.94; RMSEA $95 \%[\mathrm{CI}]=.05 \quad[.04-.06])$ como para mujeres $\left(\chi^{2}=688.6 ; \quad\right.$ g.l. $=324 ; \quad$ CFI $=.917 ; \quad$ TLI $=.910 ;$ RMSEA 95\%[CI]=.071 [.063-.078]); no obstante, se observó un mejor ajuste en el grupo de hombres que de mujeres $(\Delta \mathrm{CFI}=.034)$. La consistencia interna de la escala para la muestra total fue elevada $(\alpha=.92)$.

La edad no mostró correlación con la puntuación total en ASA-27 $(r=.08 ; p=.12)$. Respecto a la validez convergente, las correlaciones fueron elevadas y estadísticamente 
Tabla 3. Coeficientes de correlación entre ASA-27 y los diferentes cuestionarios y sus subescalas

\begin{tabular}{|c|c|c|c|c|c|c|c|c|c|c|c|c|}
\hline & \multirow[t]{2}{*}{ ASA-27 } & \multicolumn{2}{|c|}{ STAI } & \multicolumn{5}{|c|}{ CAA } & \multicolumn{4}{|c|}{$\mathrm{CDE}$} \\
\hline & & Estado & Rasgo & Total & B.A. & R.H. & E.S. & A.E. & Total & A.S. E.A.P & M.P. & M.S. E.L. \\
\hline \multicolumn{13}{|l|}{ STAI } \\
\hline Estado & .04 & & & & & & & & & & & \\
\hline Rasgo & $.52 * *$ & $.60 * *$ & & & & & & & & & & \\
\hline \multicolumn{13}{|l|}{ CAA } \\
\hline Puntuación total & $.41^{* *}$ & $.39 * *$ & $.55^{* *}$ & & & & & & & & & \\
\hline Baja autoestima & $.44 * *$ & $.39 * *$ & $.61^{* *}$ & $.87 * *$ & & & & & & & & \\
\hline Resolución hostil & $.37 * *$ & $.34 * *$ & $.45^{* *}$ & $.81 * *$ & $.60^{* *}$ & & & & & & & \\
\hline Expresión sentimientos & -.05 & -.09 & -.14 & $.30 * *$ & $.10^{*}$ & .01 & & & & & & \\
\hline Autosuficiencia emocional & $.18^{* *}$ & $.26^{* *}$ & $.30 * *$ & $.63 * *$ & $.45^{* *}$ & $.36 * *$ & .04 & & & & & \\
\hline \multicolumn{13}{|l|}{$\mathrm{CDE}$} \\
\hline Puntuación total & $.68^{* *}$ & $.31 * *$ & $.53^{* *}$ & $.48^{* *}$ & $.50 * *$ & $.45^{* *}$ & .00 & $.13^{* *}$ & & & & \\
\hline Ansiedad separación & $.65^{* *}$ & $.33^{* *}$ & $.51 * *$ & $.43 * *$ & $.49^{* *}$ & $.38 * *$ & -.03 & $.12 *$ & .92 & & & \\
\hline Expresión afectiva pareja & $.55^{* *}$ & $.27 * *$ & $.45^{* *}$ & $.45^{* *}$ & $.46^{* *}$ & $.41 * *$ & .09 & .08 & .84 & $.72 * *$ & & \\
\hline Modificación planes & $.42 * *$ & $.14^{* *}$ & $.30 * *$ & $.29 * *$ & $.27 * *$ & $.31 * *$ & -.01 & .06 & .75 & $.60^{* *} .51^{* *}$ & & \\
\hline Miedo soledad & $.59 * *$ & $.27 * *$ & $.51 * *$ & $.38 * *$ & $.39 * *$ & $.35^{* *}$ & .02 & $.12 *$ & .75 & $.65^{* *} .53^{* *}$ & $.49 * *$ & \\
\hline Expresión límite & $.54 * *$ & $.26^{* *}$ & $.43 * *$ & $.35^{* *}$ & $.38 * *$ & $.35^{* *}$ & $-.11 *$ & $.16^{* *}$ & .70 & $.58 * * .50 * *$ & $.49 * *$ & $.57 * *$ \\
\hline Búsqueda atención & $.54 * *$ & $.20 * *$ & $.39 * *$ & $.39 * *$ & $.41 * *$ & $.38 * *$ & -.05 & $.16^{* *}$ & .80 & $.66^{* *} .61 * *$ & $.53 * *$ & $.52 * * .80 * *$ \\
\hline
\end{tabular}

Nota. ASA-27 = Cuestionario de Ansiedad por Separación en el Adulto; STAI = Inventario de Ansiedad Estado-Rasgo; CAA = Cuestionario de Apego Adulto (CAA); CDS = Cuestionario de Dependencia Emocional (CDE); B.A. = Baja autoestima; R.H. = Resolución hostil; E.S. = Expresión de sentimientos; A.E. = Autosuficiencia emocional; A.S. = Ansiedad por separación; E.A.P. = Expresión afectiva pareja; M.P. Modificación de planes; M.S. = Miedo a la soledad; E.L. Expresión límite; B.A. = Búsqueda de atención.

$* p<.05, * * p<.01$

significativas en la mayoría de los casos (ver Tabla 3). Las correlaciones más elevadas entre la escala ASA-27 y el resto de las escalas y subescalas fueron para la puntuación total en la escala CDE ( $r=.68 ; p<.001)$, y para sus subescalas de «ansiedad por separación» $(r=.65 ; p<.001)$ y «miedo a la soledad» $(r=.59 ; \quad p<.001)$. No obstante, no se halló correlación entre ASA-27 y STAI-E $(r=.04 ; p=.39) ; \quad$ tampoco se halló correlación con la subescala «expresión de sentimientos» de la escala CAA ( $r=-.05 ; p=.35)$.

Se llevaron a cabo análisis de regresión múltiple incluyendo las puntuaciones en las subescalas estandarizadas como variables predictoras, y el resultado en la escala ASA-27 en su versión en español como variable dependiente. El resultado de los análisis de regresión entre las puntuaciones en la escala STAI mostró que sólo una puntuación elevada en la subescala STAIRasgo predecía una puntuación elevada en ASA$27(\beta=.62 ; p<.001)$, una vez que se controlaba la puntuación en STAI-Estado $(\beta=-.08 ; p=.14)$. Los resultados de los análisis de regresión múltiple entre las escalas CAA, CDE y ASA-27, se detallan en la Tabla 4. Respecto a las escalas
CAA, los índices «puntuación total», «expresión de sentimientos» y «autosuficiencia emocional» predecían la puntuación total en ASA-27. Para la escala CDE, los valores que predecían la puntuación en ASA-27 fueron la «puntuación total», y las subescalas «ansiedad por separación», «miedo a la soledad»y «modificación de planes». Para ambas escalas, las puntuaciones totales predijeron las puntuaciones en el ASA-27, siendo la escala CAA la que mayor valor predictor mostraba.

Los resultados mostraron diferencias significativas en las puntuaciones medias en la escala al comparar los grupos según tratamiento [t(367)=-2.57; $p<.05]$, obteniendo una puntuación media mayor el grupo que estaba bajo tratamiento psiquiátrico $(M=29, D T=16.98)$, que el grupo que no estaba bajo tratamiento $(M=15.66 ; D T=11.46)$; también se hallaron diferencias significativas $[t(364)=-3.19 ; p<.05]$ entre los que estaban bajo tratamiento psicológico $(M=25.8, \quad D T=15.69)$ comparado con los que no lo estaban $(M=15.66$; $D T=11.46)$. No se hallaron diferencias significativas para el resto de las variables. 
Tabla 4. Efecto de las puntuaciones en las subescalas Cuestionario de Apego Adulto y Cuestionario de Dependencia Emocional sobre la puntuación de ASA-27 en su versión en español (N=403)

\begin{tabular}{|c|c|c|c|c|c|c|}
\hline & \multicolumn{5}{|c|}{ Cuestionario de Apego Adulto $\beta(E E)$} & \\
\hline & Total & B.A. & R.H. & E.S. & A.E. & \\
\hline \multirow[t]{3}{*}{ ASA-27 } & $.96(.29)^{*}$ & $-.09(.32)$ & $-.25(.31)$ & $\begin{array}{c}-.31 \\
(.27)^{*}\end{array}$ & $\begin{array}{c}-.28 \\
(.31)^{*}\end{array}$ & \\
\hline & \multicolumn{5}{|c|}{ Cuestionario de Dependencia Emocional $\beta(E E)$} & \\
\hline & Total & A.S. & E.A.P & M.P. & M.S. & E.L. \\
\hline ASA-27 & $.33(.10)^{* * *}$ & $.11(.13)^{*}$ & $-.04(.14)$ & $\begin{array}{c}.23 \\
(14)^{* * *}\end{array}$ & $.14(.38)^{*}$ & $.02(.22)$ \\
\hline
\end{tabular}

Nota. ASA-27 = Cuestionario de Ansiedad por Separación en el Adulto; B.A.= Baja autoestima; R.H.= Resolución hostil; E.S.= Expresión de sentimientos; A.E.= Autosuficiencia emocional; A.S.= Ansiedad por separación; E.A.P. = Expresión afectiva pareja; M.P.= Modificación de planes; M.S.= Miedo a la soledad; E.L.= Expresión límite; B.A.= Búsqueda de atención. $* p<.05, * * p<.01, * * * p<.001$

La baremación fue conjunta para hombres y mujeres al no haberse encontrado diferencias entre ellos. De esta forma, para su aplicación clínica podríamos considerar la ausencia de problemas de ansiedad por separación en casos con un percentil por debajo de 27 (directa 8), problemas leves, percentil menor de 57 (directa 15), problemas clínicos desde percentil 60 (directa 16), y, problemas graves que requieren atención clínica, a partir de percentil 81 (directa 25). La versión española del ASA-27 se puede consultar en el Anexo 1.

\section{Discusión}

Para el estudio psicométrico del Cuestionario de Ansiedad por Separación en el Adulto (ASA27), se ha realizado la traducción y adaptación del cuestionario original a nuestro idioma, así como la traducción inversa al idioma original en inglés. Posteriormente se realizó el estudio psicométrico de fiabilidad y validez, presentando una excelente consistencia interna $(\alpha=0.92)$. Además se obtuvieron altas correlaciones con otro tipo de instrumentos empleados para una posible evaluación de este trastorno, proporcionando una excelente validez convergente.

En relación con los datos obtenidos, podemos afirmar que este instrumento es fiable y válido para la evaluación de esta problemática en población española, obteniéndose un único factor como en el cuestionario original (Manicavasagar et al., 2003). Adicionalmente se ha obtenido un baremo en percentiles considerando en conjunto hombres y mujeres, al no presentar diferencias significativas entre ambos, permitiendo determinar la gravedad de los casos en función de su puntuación, y poder utilizar el cuestionario en el proceso de diagnóstico.

En las comparaciones entre grupos encontramos diferencias significativas entre tratamiento psiquiátrico y psicológico, en comparación al grupo sin tratamiento. Esto concuerda con los estudios de Gesi et al. (2017) en relación con la búsqueda de ayuda profesional. Por otra parte, no se encontraron diferencias en las comparaciones entre edad, sexo, nivel de estudios ni trastorno o no trastorno.

Las puntuaciones obtenidas en ansiedad por separación en el adulto mediante ASA-27, tras los análisis de regresión realizados, han mostrado elevada relación con diferentes medidas de apego y dependencia emocional, encontrando mayor relación con las subescalas de «miedo a la soledad»y de «ansiedad por separación» del cuestionario CDE y con la puntuación total de este mismo cuestionario que valora de forma global dependencia emocional. Estos resultados presentan coherencia con los estudios realizados hasta el momento sobre ansiedad por separación, ya que parte de la sintomatología relevante de este cuadro tiene que ver con el miedo a no estar en contacto con el contexto conocido y sus figuras de apego, el miedo al abandono, los cambios en la rutina diaria y la soledad que experimentan estas personas.

En cuanto a las limitaciones del estudio debemos considerar la escasa muestra clínica que conforma esta investigación, teniendo como perspectiva futura aumentar esta. Así como mejorar la metodología por muestreo de bola de nieve, que a pesar de ser un método útil para 
acceder a población difícilmente accesible ofrece un control poco exhaustivo sobre la muestra. Igualmente, se podrían facilitar datos más concluyentes que permitieran facilitar baremos para población clínica y a su vez ayudasen a determinar posibles diferencias en este tipo de poblaciones, así como entre diferentes trastornos clínicos y la posible mediación de la ansiedad por separación en su desarrollo o mantenimiento. Así mismo se podría homogeneizar aún más la muestra en lo referente al sexo, la edad y otras variables demográficas, que nos permitan teniendo en cuenta los datos del Instituto Nacional de Estadística (INE, 2018), mejorar la validez externa del cuestionario. No obstante, estas variables no presentaron diferencias significativas.

Esperamos que el ASA-27 facilite el diagnóstico de TASA como instrumento específico de detección en español. Además, el cuestionario ASA-27 se ha empleado en muchos estudios clínicos en inglés, relacionado con otros trastornos donde la ansiedad por separación es relevante. Por tanto, podría ser también de gran utilidad para los clínicos en la evaluación de otros casos, viendo mejorada su intervención psicológica al considerar aspectos emocionales como la ansiedad por separación, que habitualmente no se tienen en cuenta al abordar otros tipos de problemas de ansiedad, depresivos, etc. Milrod et al. (2016) detectaron que personas con trastornos de ansiedad, que cursaban además con TASA, eran resistentes a los tratamientos psicológicos debido al no reconocimiento de este diagnóstico específico de ansiedad por separación. Por tanto, detectar adecuadamente estos problemas ha de ser un objetivo importante para mejorar los tratamientos y su efectividad, para solucionar los problemas de las personas.

\section{Referencias}

American Psychiatric Association (APA) (2014). Manual diagnóstico y estadístico de los trastornos mentales (DSM-5), $5^{a}$ Ed. Madrid: Editorial Médica Panamericana.

Baldwin, D., Gordon, R., Abelli, M., \& Pini, S. (2016). The separation of adult separation anxiety disorder. CNS Spectrums, 21, 289294. doi:10.1017/S1092852916000080
Bentler, P. M., \& Bonett, D. G. (1980). Significance tests and goodness of fit in the analysis of covariance structures. Psychological Bulletin, 88(3), 588-606. dx.doi.org/10.1037/0033-2909.88.3.588

Bögels, S., Knappe, S., \& Clark, L.A. (2013). Adult separation anxiety disorder in DSM-5. Clinical Psychology Review, 33, 663-674. doi.org/10.1016/j.cpr.2013.03.006

Boyce, P., \& Parker, G. (1989). Development of a scale to measure interpersonal sensitivity. Australian \& New Zealand Journal of Psychiatry, 23, 341-351.

Browne, M. W., \& Cudeck, R. (1992). Alternative ways of assessing model fit. Sociological Methods \& Research, 21(2), 230-258. doi.org/10.1177/0049124192021002005

Bruschi, A., De Angelis, A., Grandinetti, P., Pastucci, M., Janiri, L., \& Pozzi, G. (2012). Disturbo d'ansia di separazione dell'adulto: Evidenze di letteratura e implicazioni cliniche. Rivista di psichiatria, 47(5), 355-364. doi:10.1708/1175.13026

Carmassi, C., Gesi, C. Massimetti, E., Shear, M. K., \& Dell'Osso, L. (2015a). Separation anxiety disorder in the DSM-5 era. Journal of Psychopathology, 21, 365-371.

Carmassi, C., Gesi, C., Corsi, M., Pergentini, I., Cremone, I. M., Conversano, C., ...\& Dell'Osso, L. (2015b). Adult separation anxiety differentiates patients with complicated grief and/or major depression and is related to lifetime mood spectrum symptoms. Comprehensive Psychiatry, 58, 4549. dx.doi.org/10.1016/j.comppsych.2014.11.012

Costa, B., Pini, S., Baldwin, D. S., Silove, D., Manivasagar, V., ...\& Martini, C. (2017). Oxytocin receptor and G-protein polymorphisms in patients wit depression and separation anxiety. Journal of Affective Disorders, 218, 365-373. doi.org/10.1016/j.jad.2017.03.056

Cyranowski, J. M., Shrear, M. K., \& Rucci, P. (2002) Adult separation anxiety: Psychometric properties of a new structured clinical interview. Journal of Psychiatric Research, 36, 77-86. doi.org/10.1016/S0022-3956(01)00051-6

Dell'Osso, L., Carmassi, C., Corsi, M., Pergentini, I., Socci, C., Maremmani, A., \& Perugi, G. 
(2011). Adult separation anxiety in patients with complicated grief versus healthy control subjects: Relationships with lifetime depressive and hypomanic symptoms. Annals of General Psychiatry, 10, 29. doi:10.1186/1744-859X-10-29.

Dirioz, M., Alkin, T., Yemez, B., Onur, E., \& Eminagaoglu, B. (2011). The validity and reliability of Turkish version of Separation Anxiety Symptom Inventory and Adult Separation Anxiety Questionnaire. Turkish Journal of Psychiatry, 108-116.

Feeney, J., Noller, P., \& Hanrahan, M. (1994). Assessing adult attachment. In M. Sperling \& W. Berman (eds). Attachment in Adults (128152). New York, NY: Guilford Press.

Fernández-Marcos, T. (2014). Un caso de dolor de estómago y ansiedad por separación en una estudiante universitaria: La relevancia de la funcionalidad en la explicación del problema. Póster presentado en XII Encuentro SPPU. Valencia: Universidad Católica.

George, C., Kaplan, N., \& Main, M. (1985) The Berkeley Adult Attachment Interview. Unpublished protocol. Berkeley, CA: Department of Psychology, University of California.

Gesi, C., Abelli, M., Cardini, A., Lari, L., Di Paolo, L., Silove, D., \& Pini, S. (2015). Separation anxiety disorder from the perspective of DSM-5: Clinical investigation among subjects with panic disorder and associations with mood disorders spectrum. CNS Spectrums, 1-6. doi: 10.1017/S1092852914000807

Gesi, C., Carmassi, C., Shear, K. M., Schwartz, T., Ghesquiere, A., Khaler, J., \& Dell'Osso, L. (2017). Adult separation anxiety disorder in complicated grief: An exploratory study of frequency and correlates. Comprehensive Psychiatry, 72, 6-12. doi.org/10.1016/j.comppsych.2016.09.002

González, R., Ysern, L., Martorell, C., Matéu, C., \& Barreto, P. (2011). Relaciones entre psicopatología y apego en la adolescencia. Revista Iberoamericana de Diagnóstico y Evaluación - e Avaliação Psicológica, 25(1), 107-125.

INE. (2018). España en cifras. Madrid: Instituto Nacional de Estadística
Kessler, R. C., Petukhova, M., Sampson, N. A., Zaslavsky, A. M., \& Wittchen, H. U. (2012). Twelve-month and lifetime prevalence and lifetime morbid risk of anxiety and mood disorders in the United States. International journal of methods in psychiatric research, 21(3), 169-184. doi:10.1002/mpr.1359

Kessler, R. C., \& Ustun, T. B. (2004). The World Mental Health (WMH) survey initiative version of the World Health Organization (WHO) Composite International Diagnostic Interview (CIDI). International Journal of Methods in Psychiatric Research, 13, 93-121.

Kossowsky, J., Pfaltz, M. C., Schneider, S., Taeymans, J., Locher, C., \& Gaab, J. (2013). The separation anxiety hypothesis of panic disorder revisited: A meta-analysis. The American Journal of Psychiatry, 170(7), 768781. doi.org/10.1176/appi.ajp.2012.12070893

Lemos, M. A., \& Londoño, N. H. (2006). Construcción y validación del cuestionario de dependencia emocional en población colombiana. Acta Colombiana de Psicología, 9(2), 127-140.

Mabilia, D., Di Riso, D., Liso, A., \& Bobbio, A. (2019). A prediction model for sepration anxiety: The role of attachment styles and internalizing symptoms in Italian young adults. Journal of Adult Developmet. 1-9. doi.org/10.1007/s10804-019-09327-y

Manicavasagar, V., \& Silove, D. (1997). Is there an adult form of separation anxiety disorder? A brief clinical report. Australian \& New Zealand Journal of Psychiatry, 31, 299-303.

Manicavasagar, V., \& Silove, D. (2016). Why are we still missing adult separation anxiety disorder in clinical practice? Australian \& New Zealand Journal of Psychiatry, 50(8), 807-808.

Manicavasagar, V., Silove, D. Wagner, R., \& Drobney, J. (2003). A self-report questionnaire for measuring separation anxiety in adulthood. Comprehensive Psychiatry, 44(2), 146-153. doi.org/10.1053/comp.2003.50024

Manicavasagar, V., Silove, D., Curtis, J., \& Wagner, R. (2000). Continuities of separation anxiety from early life into adulthood. Journal of Anxiety Disorders, 4, 1-18. doi.org/10.1016/S0887-6185(99)00029-8 
Manicavasagar, V., Silove, D., Wagner, R., \& Drobny, J. (2012). Adult Separation Anxiety Questionnaire - ASA-27. Measurement instrument Database for the Social Science. Retrieved from www.midss.ie. dx.doi.org/10.13072/midss.162

Manivacasagar, V., Marnane, C., Pini, S., Abelli, M., Rees, S., Eapen, V., \& Silove, D. (2010). Adult separation anxiety disorder: A disorder comes of age. Current Psychiatry Report, 12, 290-297.

Melero, R., \& Cantero, M. J. (2008). Los estilos afectivos en la población española: Un cuestionario de evaluación del apego adulto. Clínica y Salud, 19(1), 83-100.

Milrod, B., Altemus, M., Gross, C., Busch, F., Silver, G., Christos, P., ...\& Schneier, F. (2016). Adult separation anxiety in treatment nonresponders with anxiety disorders: Delineation of the syndrome and exploration of attachment-based psychotherapy and biomarkers. Comprehensive Psychiatry, 66, 139-145.

doi.org/10.1016/j.comppsych.2016.01.004

Milrod, B., Markowitz, J. C., Gerber, A. J., Cyranowski, J., Altemus, M., Shapiro, T., ...\& Glatt, C. (2014). Childhood separation anxiety and the pathogenesis and treatment of adult anxiety. American Journal of Psychiatry, 171(1), 34-43. doi:10.1176/appi.ajp.2013.13060781.

Möller, E. L., \& Bögels, S. M. (2016). The DSM5 Dimensional Anxiety Scales in a Dutch nonclinical sample: Psychometric properties including the adult separation anxiety disorder scale. International Journal of Methods in Psychiatric Research, 25(3), 232-239. doi:10.1002/mpr.1515

Nunnally, J. C. (1978). Psychometric Theory (2nd edition). New York: Mc-Graw-Hill.

Perugi, G., Toni, C., Maremmani, I., Tusini, G., Ramacciotti, S., Madia, A., ...\& Akiskal, H.S. (2012). The influence of affective temperaments and psychopathological traits on the definition of bipolar disorders subtypes: A study on Bipolar I Italian national sample. Journal of Affective Disorders, 136, 41-49. doi:10.1016/j.jad.2009.12.027

Pini, S., Abelli, M., \& Bruschi, A. (2014a). Separation anxiety disorder in adulthood. In
R. Guglielmo, L. Janiri, \& G. Pozzi (eds.). New perspectives on generalized anxiety disorder (pp. 149-167). New York: Nova Science Publishers, Inc.

Pini, S., Abelli, M., Mauri, M., Muti, M., Lazzetta, P., Banti, S., \& Cassano, G. B. (2005). Clinical correlates and significance of separation anxiety in patients with bipolar disorder. Bipolar Disorders, 7(4), 370-376. doi: 10.1111/j.1399-5618.2005.00216.x

Pini, S., Abelli, M., Shear, K. M., Cardini, A., Lari, L., Gesi, C., ...\& Cassano, G. B. (2010). Frequency and clinical correlates of adult separation anxiety in a sample of 508 outpatients with mood and anxiety disorders. Acta Psychiatrica Scandinavica, 122, 40-6. doi:10.1111/j.1600-0447.2009.01480.x

Pini, S., Abelli, M., Troisi, A., Siracusano, A., Cassano, G. B., Shear, K. M., \& Baldwin, D. (2014b). The relationships among separation anxiety disorder, adult attachment style and agoraphobia in patients with panic disorder. Journal of Anxiety Disorders, 28, 741-746. dx.doi.org/10.1016/j.janxdis.2014.06.010

Posavac, S. S., \& Posavac, H. D. (2017). Adult separation anxiety disorder symptomology and susceptibility to marketing persuasion. Journal of Social and Clinical Psychology, $36(2)$, 158-169. doi.org/10.1521/jscp.2017.36.2.158

Pozzi, G., Bruschi, A., De Angelis, A., Pascucci, M., Hatzigiakoumis, D. S., Grandinetti, P., ...\& Janiri, L. (2014). Adult separation anxiety and TCI-R personality dimensions in patients with anxiety, alcohol use, and gambling: A preliminary report. Biomed Research International.

doi:dx.doi.org/10.1155/ 2014/680985

Román, M., Hodges, J., Palacios, J., Moreno, C., \& Hillman, S. (2015). Evaluación de las representaciones mentales de apego a través de historias incompletas: Aplicación española de Story Stem Assessment Profile (SSAP). Revista Iberoamericana de Diagnóstico y Evaluación - e Avaliação Psicológica, 46(1), 5-19. doi.org/10.21865/RIDEP46.1.01

Ruiz, A., \& Rando, M.A. (2015). Tratamiento de un caso de ansiedad por separación en una mujer adulta. Póster presentado en XII 
Encuentro SPPU. Madrid: Universidad Carlos III.

Schneier, F. R., Moskow, D. M., Choo, T-H., Galfalvy, H., Campeas, R., \& Sanchez-Lacay, A. (2017). A randomized controlled pilot trial of vilazodone for adult separation anxiety disorder. Depress Anxiety. 1-11. doi:10.1002/da.22693

Shear, K., Jin, R., Ruscio, A. M., Walters, E. E., \& Kessler, R. C. (2006). Prevalence and correlates of estimated DSM-IV child and adult separation anxiety disorder in the National Comorbidity Survey Replication. The American Journal of Psychiatry, 163, 1074-1083.

Silove, D., \& Rees, S. (2014). Separation anxiety disorder across the lifespan: DSM-5 lifts age restriction on diagnosis. Asian Journal of Psychiatry, 11, 98-101. doi: 10.1016/j.ajp.2014.06.021

Silove, D., Alonso, J., Bromet, E., Gruber, M., Sampson, N., Scott, K., ...\& Kessler, R.C. (2015). Pediatric-onset separation and Adultonset Separation anxiety disorder across countries in the World Mental Health Survey. The American Journal of Psychiatry, 172, 647656. doi:10.1176/appi.ajp.2015.14091185

Silove, D., Manicavasagar, V., O'Connell, D., Blaszczynski, A., Wagner, R., \& Henry, J. (1993). The development of the separation anxiety symptom inventory (SASI). Australian and New Zealand Journal of Psychiatry, 27, 477-488.

Silove, D., Marnane, C., Wagner, R., \& Manicavasagar, V. (2011). Associations of personality disorder with early separation anxiety in patients with adult separation anxiety disorder. Journal of Personality Disorders, 25, 128-33.

Silove, D., Slade, T., Marnane, C., Wagner, R., Brooks, R., \& Manicavasagar, V. (2007). Separation anxiety in adulthood: Dimensional or categorical? Comprehensive Psychiatry, $48(6)$, 546-53. doi.org/10.1521/pedi.2011.25.1.128

Silove, D. M., Marnane, C.L., Wagner, R., Manicavasagar, V. L., \& Rees, S. (2010). The prevalence and correlates of adult separation anxiety disorder in an anxiety clinic. $B M C$
Psychiatry,

10 , $10-21$.

doi.org/10.1016/j.comppsych.2007.05.011

Spielberger, C. D., Gorsuch, R. L., \& Lushene, R. E. (1994). Cuestionario de Ansiedad EstadoRasgo. Madrid: TEA. 


\section{ANEXO 1}

\section{Cuestionario de Ansiedad en el Adulto (ASA-27)}

(Adaptación de Manicavasagar, Silove, Wagner \& Drobny, 2003)

Los siguientes estados se refieren a características emocionales que podría haber experimentado en la edad adulta (a partir de los 18 años de edad). Por favor, marque la casilla correspondiente con una cruz para cada ítem, si ha experimentado alguno de esos síntomas. Por favor, recuerde responder a todas las preguntas.

\section{No me ha sucedido nunca \\ 1 Me sucede ocasionalmente \\ 2 Me sucede bastante a menudo \\ 3 Me sucede muy a menudo}

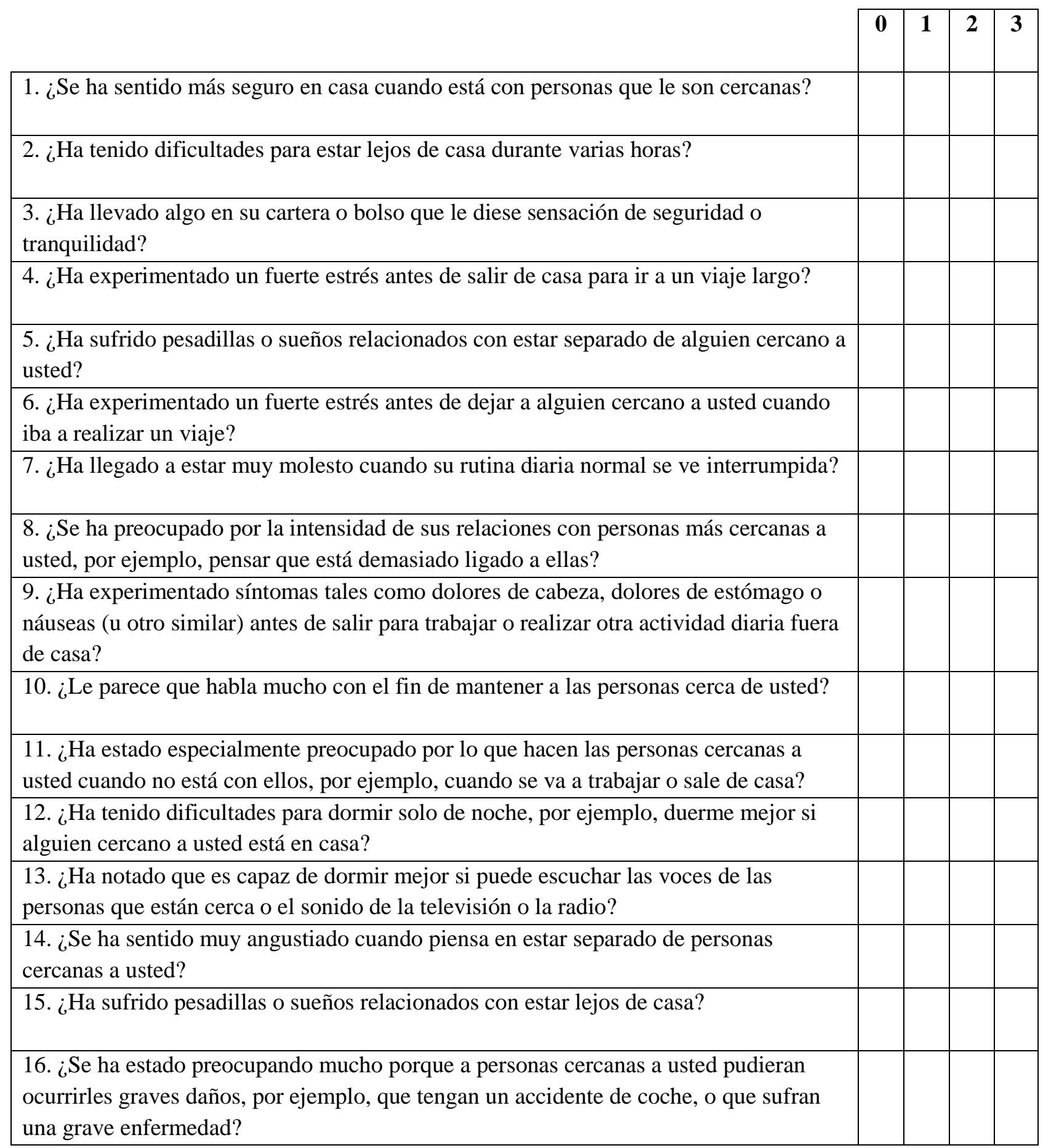


17. ¿Han llegado a molestarle mucho los cambios en su rutina diaria normal si eso interfiere en su contacto con las personas cercanas a usted?

18. ¿Se ha estado preocupando mucho porque las personas que le importan le dejen?

19. ¿Ha encontrado que duerme mejor si las luces de casa o las del dormitorio están encendidas?

20. ¿Ha tratado de evitar quedarse solo en casa, especialmente cuando las personas cercanas a usted estaban fuera?

21. ¿Ha sufrido de ataques repentinos de ansiedad o ataques de pánico (por ejemplo temblores súbitos, sudoración, dificultad para respirar, taquicardia) cuando piensa en dejar a personas que le importan o que éstas le dejen?

22. ¿Ha encontrado que se siente ansioso si no habla por teléfono con regularidad con las personas que le son cercanas, por ejemplo, todos los días?

23. ¿Ha tenido miedo de no ser capaz de afrontar o seguir adelante, si alguien que le importa le dejara?

24. ¿Ha sufrido de ataques repentinos de ansiedad o ataques de pánico (por ejemplo temblores súbitos, sudoración, dificultad para respirar, taquicardia) cuando se ha separado de personas cercanas a usted?

25. ¿Se ha estado preocupando mucho de posibles acontecimientos que pudieran separarle de aquellos que le son cercanos, por ejemplo, debido a obligaciones del trabajo?

26. ¿La gente cercana a usted le ha dicho que usted "habla mucho"?

27. ¿Le ha estado preocupando que la relación con algunas personas sea tan cercana que pueda ocasionarles problemas a ellos? 


\section{ANEXO 2}

Tabla de puntuaciones directas y percentiles correspondientes

\begin{tabular}{cccc}
\hline Percentil & P. Directa & Percentil & P. Directa \\
\hline 2 & 0 & 75 & 22 \\
5 & 1 & 76 & 23 \\
8 & 2 & 79 & 24 \\
10 & 3 & 81 & $25-26$ \\
12 & 4 & 82 & 27 \\
14 & 5 & 83 & 28 \\
18 & 6 & 84 & 29 \\
21 & 7 & 85 & 30 \\
27 & 8 & 86 & 31 \\
31 & 9 & 87 & 32 \\
36 & 10 & 88 & $33-34$ \\
39 & 11 & 89 & 35 \\
45 & 12 & 90 & 36 \\
49 & 13 & 91 & 37 \\
53 & 14 & 92 & $38-40$ \\
57 & 15 & 94 & 41 \\
60 & 16 & 95 & $42-43$ \\
63 & 17 & 96 & 44 \\
65 & 18 & 97 & $45-46$ \\
67 & 19 & 98 & $47-49$ \\
70 & 20 & 99 & $50-55$ \\
72 & 21 & 100 & $61-70$ \\
$M$ & 17.03 & $D T$ & 12.55 \\
\hline & & &
\end{tabular}

